\title{
Lightweight Probabilistic Broadcast
}

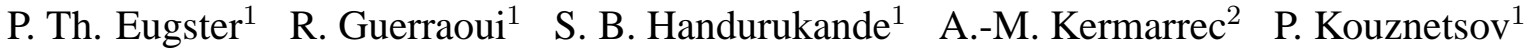 \\ ${ }^{1}$ Federal Institute of Technology, Lausanne, Switzerland \\ ${ }^{2}$ Microsoft Research, Cambridge, UK
}

\begin{abstract}
The growing interest in peer-to-peer applications has underlined the importance of scalability in modern distributed systems. Not surprisingly, much research effort has been invested in gossip-based broadcast protocols. These trade the traditional strong reliability guarantees against very good "scalability" properties. Scalability is in that context usually expressed in terms of throughput, but there is only little work on how to reduce the overhead of membership management at large scale.

This paper presents Lightweight Probabilistic Broadcast (lpbcast), a novel gossip-based broadcast algorithm which preserves the inherent throughput scalability of traditional gossip-based algorithms and adds a notion of membership management scalability: every process only knows a random subset of fixed size of the processes in the system. We formally analyze our broadcast algorithm in terms of scalability with respect to the size of individual views, and compare the analytical results both with simulations and concrete measurements.
\end{abstract}

\section{Introduction}

Large scale event dissemination. Peer-to-peer computing has recently received much attention, as shown by the success of large scale decentralized applications like Gnutella [30] or Groove [12]. In peer-to-peer computing, every process acts as client and server, and scalability is a major concern.

The scalability properties solicited from such applications have evolved from hundreds to thousands of participants, but adequate algorithms for reliable propagation of events at large scale are still lacking. Network-level protocols have turned out to be insufficient: IP multicast [6] lacks reliability guarantees, and reliable protocols do not scale well. The well-known Reliable Multicast Transport Protocol (RMTP) [24] for instance generates a fload of positive acknowledgements from receivers, loading both the network and the sender, where these acknowledgements converge. Any form of membership $([21,15,2])$ is hidden by such network-level protocols, which makes them consequently also difficult to exploit with more dynamic dissemination (filtering, e.g., [22]), emphasizing the need for new forms of application-level broadcast. 
Gossip-based broadcast algorithms. Gossip-based broadcast algorithms (e.g., [4, 26, 18]) appear to be more adequate in the field of large scale event dissemination, than the "classical" strongly reliable approaches [14]. Though such gossip-based approaches have proven good scalablility characteristics in terms of throughput, they often rely on the assumption that every process knows every other process. When managing large numbers of processes, i.e., a large number of references to processes acting as event producers and/or consumers, this assumption becomes a barrier to scalability. In fact, the data structures necessary to store the view of such a large scale membership consume considerable memory resources, let aside the communication required to ensure the consistency of the membership.

Partial view. Message routing and membership management are sometimes delegated to dedicated servers ${ }^{1}$ in order to relief application processes. This only defers the problem, since those servers are limited in resources as well. To further increase scalability, the membership view should be split, i.e., every participating process should only dispose of a partial view of the system. In order to avoid the isolation of processes or the partition of the membership, especially in the case of failures, membership information should nevertheless be shared by processes to some extent: introducing a certain degree of redundancy between the individual views is crucial to avoid single points of failure.

Gossip-based membership. While certain systems rely on a deterministic scheme to establish the individual views $[28,18]$, we introduce here a new completely randomized approach. The local view of every individual member consists in a random process list which continuously evolves, but never exceeds a fixed size. In short, after adding new processes to a view, it is truncated to the maximum length by removing randomly chosen entries. To ensure a uniform distribution of membership knowledge among processes, every gossip message - besides notifying events mainly also piggybacks a set of process identifiers which are used to update views. The membership protocol and the effective dissemination of events are thus dealt with at the same level. This symmetry is precisely the key to our formal analysis.

Contributions. We present in this paper our strongly scalable decentralized algorithm for event dissemination, called

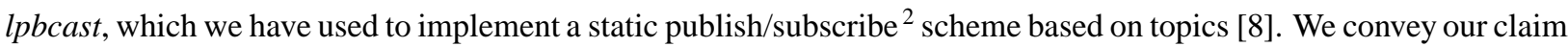
of scalability in two steps. First, we formally analyze our algorithm using a stochastic approach, pointing out the fact that, with perfectly uniformly distributed individual views, the view size has no impact on the latency of delivery of an event. We similarly show that for a given view size, the probability of partition creation in the system decreases as the system grows in size. Second, we give some practical results that support the analytical approach, both in terms of simulation and prototype measurements.

It is important to notice that our membership approach is not intrinsically tied to our Lightweight Probabilistic Broadcast (lpbcast) algorithm. We illustrate this by applying our membership scheme to the well-known pbcast [4] algorithm.

Roadmap. Section 2 gives an overview of related gossip-based broadcast protocols. Section 3 presents our lpbcast algorithm and explains our randomized approach. Section 4 presents a formal analysis of our algorithm in terms

\footnotetext{
${ }^{1}$ These are also called event servers [5], routing daemons [27], or message brokers [1].

${ }^{2}$ Due to its decoupling nature, the publish/subscribe paradigm has been used in various large scale contexts, e.g, [20, 9].
} 
of scalability and reliability. Section 5 gives some simulation and practical results supporting the formal analysis. Section 6 discusses the distribution of the views and also proves the general applicability of our membership approach by combining it with pbcast and contrasting the consolidated algorithm with lpbcast. Section 7 concludes the paper.

\section{Background: Probabilistic Algorithms}

The achievement of strong reliability guarantees (in the sense of [14]) in practical distributed systems requires expensive mechanisms to detect missing messages and initiate retransmissions. Due to the overhead of message loss detection and reparation, protocols offering such strong guarantees do not scale over a couple of hundred processes [25].

\subsection{Reliability vs Scalability}

Gossip, or rumor mongering algorithms [7], are a class of epidemiologic algorithms, which have been introduced as an alternative to such "traditional" reliable broadcast protocols. They have first been developed for replicated database consistency management [7]. The main motivation is to trade the reliability guarantees offered by costly deterministic protocols against weaker reliability guarantees, but in return obtain very good scalability properties.

Their analysis is usually based on stochastics similar to the theory of epidemics [3], where the execution is broken down in steps. Probabilities are associated to these steps, and such algorithms are therefore sometimes also referred to as probabilistic algorithms. The degree of reliability is typically expressed by a probability; like the probability 1- $\alpha$ of reaching all processes in the system for any given message, or by a probability $1-\beta$ of reaching any given process with any given message. Ideally, $\alpha$ resp. $\beta$ are precisely quantifiable.

\subsection{Basic Concepts}

Decentralization is the key concept underlying the scalability properties of gossip-based broadcast algorithms, i.e., the overall load of retransmissions is reduced by decentralizing the effort. In contrast to sender-reliable protocols (e.g., Reliable Multicast Transport Protocol (RMTP) [24]) or receiver-reliable protocols (e.g., Log-Based ReceiverReliable Multicast (LBRM) [16]), ${ }^{3}$ gossip-based broadcast protocols are part of the class of peer-based protocols, just like Scalable Reliable Multicast (SRM) [10]. While retransmission requests in SRM can be handled by any process but lead to the re-broadcasting of a message, gossip-based protocols abide even better to the nature of peerto-peer computing, by relying on pairwise interaction between peers. More precisely, retransmissions are initiated in most gossip-based algorithms by having every process periodically (every $T$ ms - step interval) send a digest of the messages it has delivered to a randomly chosen subset of processes inside the system (gossip subset). The size of the subset is usually fixed, and is commonly called fanout $(F)$. Gossip protocols differ in the number of times the same information is gossiped, i.e., every process might gossip the same information only a limited number of times (repetitions are limited) and/or the same information might be forwarded only a limited number of times (hops are limited).

\footnotetext{
${ }^{3}$ In the first class of protocols (e.g. RMTP), senders wait for acknowledgements from receivers, while in the second class (e.g., LBRM), receivers are responsible for detecting missing messages and soliciting retransmissions from senders.
} 


\subsection{Membership Tracking in Gossip-Based Algorithms}

Membership tracking in gossip-based algorithms is a challenging issue. Early approaches like [11] admit that the individual views of processes diverge temporarily, but assume that they eventually converge in "stable" phases. These views however represent the "complete" membership, which becomes a bottleneck at an increased scale. The Bimodal Multicast [4] and Directional Gossip [18] algorithms are representatives of a new generation of probabilistic algorithms - aware of the problem of scalable membership management.

Bimodal Multicast. Bimodal Multicast (also called pbcast) relies on two phases. A "classical" best-effort multicast protocol (e.g., IP multicast) is used for a first rough dissemination of messages. A second phase assures reliability with a certain probability, by using a peer-based retransmission based on gossips: ${ }^{4}$ every process in the system periodically gossips a digest of its received messages, and gossip receivers can solicit such messages from the sender if they have not received them previously. ${ }^{5}$

In [4], the membership problem is not dealt with, but the authors refer to another paper which deals with failure detection based on gossips [29], while a third paper describes Capt'n Cook [28], a gossip-based resource location protocol for the Internet, which can in that sense be seen as a membership protocol. ${ }^{6}$ This protocol enables the reduction of the view of each individual process: each process has a precise view of its immediate neighbours, while the knowledge becomes less exhaustive at increasing "distance". The notion of distance is expressed in terms of host addresses. [28] however only considers the propagation of membership information and it is thus not clear how this membership interacts with pbcast.

Directional Gossip. Directional Gossip is a protocol especially targeted at wide area networks. By taking into account the topology of the networks and the current processes, optimizations are performed. More precisely, a weight is computed for each neighbour node, representing the connectivity of that given node. The larger the weight of a node, the more possibilities exist thus for it to be infected by any node. The protocol applies a simple heuristic, which consists in choosing nodes with higher weights with a smaller probability than nodes with smaller weights. That way, redundant sends are reduced. The algorithm is also based on partial views, in the sense that there is a single gossip server per LAN which acts as a bridge to other LANs. This however leads to a static hierarchy, in which the failure of a gossip server can isolate several processes from the remaining system.

In contrast to the deterministic hierarchical membership approaches in Directional Gossip or Capt'n Cook, our lpbcast algorithm has a probabilistic approach to membership: each process has a random partial view of the system. lpbcast is light weight in the sense that it consumes little resources in terms of memory and requires no dedicated messages for membership management: gossip messages are used to disseminate notifications ${ }^{7}$ and to propagate

\footnotetext{
${ }^{4}$ In order to offer a complete guarantee of delivery, Reliable Probabilistic Multicast (rpbcast) [26] adds a deterministic third phase to the pbcast protocol, in which centralized loggers are used if the second gossip-based phase fails.

${ }^{5}$ This is commonly referred to as gossip pull in contrast to gossip push, where gossip senders are updated by gossip receivers with messages missing in the digest gossiped by the former one (rpbcast uses gossip push). The term anti-entropy usually refers to a mixed push/pull variant, where two processes symmetrically update each other.

${ }^{6}$ Gossip-based garbage collection is dealt with in [13].

${ }^{7}$ These notifications constitute the actual payload of the gossip messages, and can be viewed as application messages. In contrast, gossip messages constitute protocol messages. This distinction was not made previously, since gossips are seldom used as "primary" means of dissemination.
} 
digests of received events, but also to propagate membership information.

\section{Lightweight Probabilistic Broadcast (lpbcast)}

In this section, we present our completely decentralized lightweight probabilistic algorithm for event dissemination based on partial views. Though the parts concerning the event dissemination and the membership respectively can be considered as independent, we present our solution as a monolithical algorithm. This is done in order to simplify presentation, and to emphasize the possibility of dealing with membership and event dissemination at the same level.

\subsection{System Model}

We consider a system of processes $\Pi=\left\{p_{1}, p_{2}, \ldots\right\}$. Processes join and leave the system dynamically and have ordered distinct identifiers. We assume for presentation simplicity that there is not more than one process per node of the network.

Though our algorithm has been implemented in the context of topic-based publish/subscribe [8], we present it with respect to a single topic, and do not discuss the effect of scaling up topics. In other terms, $\Pi$ can be considered as a single topic or group, and joining/leaving $\Pi$ can be viewed as subscribing/unsubscribing from the topic. Such subscriptions/unsubscriptions are assumed to be rare compared to the large flow of events, and every process in $\Pi$ can subscribe to and/or publish events.

\subsection{Gossip Messages}

Our lpbcast algorithm is based on non-synchronized periodical gossips, where a gossip message contains several types of information. To be more precise, a gossip message serves four purposes:

Notifications: A message piggybacks notifications received (for the first time) since the last outgoing gossip message. Each process stores these notifications in a variable events. Every such notification is only gossiped at most once. Older notifications are stored in a different buffer, which is only required to satisfy retransmission requests.

Notification identifiers: Each message also carries a digest (history) of notifications that the sending process has received. To that end, every process stores identifiers of notifications it has already delivered in a variable eventIds. We suppose that these identifiers are unique, and include the identifier of the originator. That way, the buffer can be optimized by only retaining for each sender the identifiers of notifications delivered since the last one delivered in sequence.

Unsubscriptions: A gossip message also piggybacks a subset of unsubscriptions. This type of information enables the gradual removal of processes which have unsubscribed from local views. Unsubscriptions that are eligible to be forwarded with the next gossip(s) are stored in a variable unSubs.

Subscriptions: A set of subscriptions are attached to each message. These subscriptions are buffered in subs. A gossip receiver uses these subscriptions to update its view, stored in a variable view. 
Note that none of the outlined data structures contains duplicates. That is, trying to add an already contained element to a list leaves the list unchanged. Furthermore, every list has a maximum size, noted $|L|_{m}$ for a given list $L$ $\left(\forall L,|L| \leq|L|_{m}\right)$. As a prominent parameter, the maximum length of view $\left(\mid\right.$ view $\left.\left.\right|_{m}\right)$ will be denoted $l$.

\subsection{Procedures}

The algorithm is composed of two parts. The first part is executed upon reception of a gossip message, and the second part is repeated periodically in attempt to propagate information to other processes.

Gossip reception. According to the lists that are attached to each gossip message, there are several phases in the handling of an incoming message (Figure 1(a)).

I. In a first phase, the unsubscriptions are handled. Every unsubscription is applied to the local view (view), and then added to the list of potentially forwarded unsubscriptions unSubs. This list is then truncated randomly to respect the maximum size limit.

II. The second phase consists in trying to add not yet contained subscriptions to the local view. These are also eligible for being forwarded with the next outgoing gossip message. Note that the subscriptions potentially forwarded with the next outgoing gossip message, stored in subs, are a random mixture of subscriptions which are present in the view after the execution of this phase, and subscriptions removed to respect the maximum size limit of view. Finally, subs is also truncated randomly to respect the maximum size limit.

III. In this third phase, notifications which have been received for the first time with the last incoming gossip message are delivered to the application. Multiple deliveries are avoided by storing all identifiers of delivered notifications in eventIds, as previously outlined. Delivered notifications are at the same time eligible for being forwarded with the next gossip.

Gossip sending. Each process periodically (every $T \mathrm{~ms}$ ) generates a gossip message - according to Section 3.2 which it gossips to $F$ other processes, randomly chosen among the local view (view). This is done even if the process has not received any new notifications since it last sent a gossip message. In that case, gossip messages are solely used to exchange digests and maintain the views uniformly distributed. The network thus experiences little fluctuations in terms of overall load due to gossip messages, as long as the number of processes inside $\Pi$ and also $T$ remain unchanged.

\subsection{Subscribing and Unsubscribing}

For presentation simplicity we have not reported the procedures for subscribing/unsubscribing in Figure 1(a). In short, a process $p_{i}$ which wants to subscribe must know a process $p_{j}$ which is already in $\Pi$. Process $p_{i}$ will send its subscription to that process $p_{j}$, which will gossip that subscription on behalf of $p_{i}$. If the subscription of $p_{i}$ is correctly received and forwarded by $p_{j}, p_{i}$ will be gradually added to the system. Process $p_{i}$ will experience this by receiving more and more gossip messages. Otherwise, a timeout will trigger the re-emission of the subscription request. 
Process $p_{i}:$

\section{upon RECEIVE (gossip)}

\{Phase 1: Update view and unSubs with unsubscriptions\}

for all unsub $\in$ gossip.unSubs do

view $\leftarrow$ view $\backslash\{$ unsub $\}$

unSubs $\leftarrow$ unSubs $\cup\{$ unsub $\}$

while $\mid$ unSubs $|>|$ unSubs $\left.\right|_{m}$ do

remove random element from unSubs

\{Phase 2: Update view with new subscriptions\}

for all newSub $\in$ gossip.subs $\wedge$ newSub $\neq \mathrm{p}_{i}$ do

if newSub $\notin$ view then

view $\longleftarrow$ view $\cup$ newSub

subs $\leftarrow$ subs $\cup$ newSub

while $\mid$ view $\mid>l$ do

target $\leftarrow$ random element in view

view $\leftarrow$ view $\backslash\{$ target $\}$

subs $\leftarrow$ subs $\cup\{$ target $\}$

while $\mid$ subs $|>|$ subs $\left.\right|_{m}$ do

remove random element from subs

\{Phase 3: Update events with new notifications\}

for all e $\in$ gossip.events do

if e.id $\notin$ eventIds then

events $\leftarrow$ events $\cup\{\mathrm{e}\}$

LPB-DELIVER(e)

eventIds $\leftarrow$ eventIds $\cup\{$ e.id $\}$

while $\mid$ eventIds $|>|$ eventIds $\left.\right|_{m}$ do

remove oldest element from eventIds

while $\mid$ events $|>|$ events $\left.\right|_{m}$ do

remove random element from events
Process $p_{i}:$

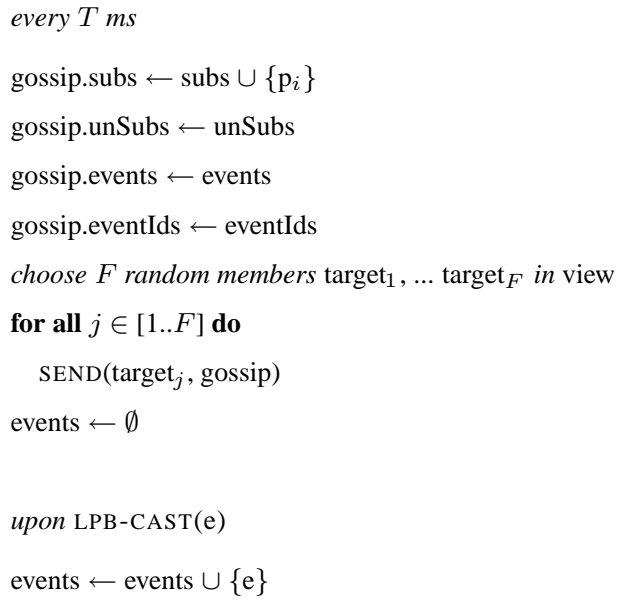

(b) Gossip emission

(a) Gossip reception

Figure 1. lpbcast algorithm 
Similarly, when unsubscribing, the process is gradually removed from local views. To avoid the situation where unsubscriptions remain in the system forever (since $u n S u b s$ is not purged), there is a timestamp attached to every unsubscription. After a certain time, the unsubscription becomes obsolete. It is important to notice that this scheme is not applied to subscriptions: these are continuously dispatched in order ensure uniformly distributed views.

Also, to avoid the removal of the unsubscription of a process from its own local unSubs (by truncating unSubs) before the unsubscription has been gossiped, the unsubscription of any process is refused as long as the local unsubscription buffer of the process exceeds a given size. This increases the probability for a process to be successfully removed from the system.

\section{Analytical Evaluation}

This section presents a formal analysis of our lpbcast algorithm. The goal is to show the impact of the size $l$ of the individual views of processes both (1) on the latency of delivery and (2) on the stability of our membership. The analysis differs from the one proposed in [4], precisely because our membership is not global and a same notification is not forwarded only a limited number of times (hops are not limited), and can be forwarded several times by the same process (repetitions are not limited). Similarly however, we first introduce a set of assumptions without which the analysis becomes extremely tedious, but which have only very little impact on its validity.

\subsection{Assumptions}

For our formal analysis we consider a snapshot of the system composed of $|\Pi|=n$ processes, and we observe the behaviour of $\Pi$ with respect to a "run", i.e., the propagation of a single event notification. We assume that the composition of $\Pi$ does not vary during the run (consequently $n$ is constant). According to the terminology applied in epidemiology, a process which has delivered a given notification will be termed infected, otherwise susceptible.

The stochastic analysis presented below is based on the assumption that processes gossip in synchronous rounds, and there is an upper bound on the network latency which is smaller than a gossip period $T . T$ is furthermore constant and identical for each process, just like $F$. We assume furthermore that failures are stochastically independent. The probability of a message loss does not exceed a predefined $\varepsilon>0$, and the number of process crashes in a run does not exceed $f<n$. The probability of a process crash during a run is thus bounded by $\tau=f / n$. For the following computations and also for the simulations in the next section, we will assume $\tau=0.01$ and $\varepsilon=0.05$. We do not take into account the recovery of crashed processes, nor do we consider byzantine (or arbitrary) failures.

At each round, each process has a uniformly distributed random view of size $l$ of known subscribers. In other terms, every combination of $l$ within $n-1$ processes $^{8}$ is equally probable for every individual view. For simplicity reasons, we will also refer to such views as uniform views (though this is a language abuse). The expected number of processes which know a given process is thus $\approx l$. As already mentioned however, these views are not constant, but continue evolving.

\footnotetext{
${ }^{8}$ According to the algorithm presented in Figure 1(a), a process $p_{i}$ will never add itself to its own local view $\left(v i e w_{i}\right)$.
} 


\subsection{Event Propagation}

Let $e$ be an event produced (LPB-CAST) by a given process. We denote the number of processes infected with $e$ at round $r$ as $s_{r} \in[1 . . n]$. Note that when $e$ is injected into the system at round $r=0$, we have $s_{r}=1$.

We define a lower bound on the probability that a given susceptible process is infected by a given gossip message as:

$$
\begin{aligned}
p & =\left(1-\frac{\left(\begin{array}{c}
n-2 \\
l
\end{array}\right)}{\left(\begin{array}{c}
n-1 \\
l
\end{array}\right)}\right)\left(\frac{F}{l}\right)(1-\varepsilon)(1-\tau) \\
& =\left(\frac{l}{n-1}\right)\left(\frac{F}{l}\right)(1-\varepsilon)(1-\tau) \\
& =\left(\frac{F}{n-1}\right)(1-\varepsilon)(1-\tau)
\end{aligned}
$$

In other terms, $p$ is expressed as a conjunction of four conditions, namely that (1) the considered process is known by the process which gossips the message, (2) the considered process is effectively chosen as target, (3) the gossip message is not lost in transit, and (4), the target process does not crash. As a direct consequence of the uniform distribution of the individual views, $p$ does not depend on $l$.

Accordingly, $q=1-p$ represents the probability that a given process is not infected by a given gossip message. Given a number $i$ of currently infected processes, we are now able to define the probability that exactly $j$ processes will be infected at the next round ( $j-i$ susceptible processes are infected during the current round). The resulting Markov Chain is characterized by the following probability $p_{i j}$ of transiting from state $i$ to state $j:^{9}$

$$
\begin{aligned}
p_{i j} & =P\left(s_{r+1}=j \mid s_{r}=i\right) \\
& = \begin{cases}\left(\begin{array}{c}
n-i \\
j-i
\end{array}\right)\left(1-q^{i}\right)^{j-i} q^{i(n-j)} & j \geq i \\
0 & j<i\end{cases}
\end{aligned}
$$

The distribution of $s_{r}$ can then be computed recursively:

$$
\begin{aligned}
& P\left(s_{0}=j\right)= \begin{cases}1 & j=1 \\
0 & j>1\end{cases} \\
& P\left(s_{r+1}=j\right)=\sum_{i \leq j} P\left(s_{r}=i\right) p_{i j}
\end{aligned}
$$

\subsection{Gossip Rounds}

By considering that the two parameters $\tau$ and $\varepsilon$ are beyond the limits of our influence, the determining factors according to the analysis are the fanout $F$ and of course the System size $n$.

\footnotetext{
${ }^{9}$ We assume here that the probability of $x$ processes infecting $y$ susceptible processes is the conjunction of the probabilities of each of the $y$ processes receiving a gossip message from at least one of the $x$ processes, and that these probabilities are independent. More precisely, we include scenarios in which the sum of considered messages sent in a round exceeds the effective sum of messages sent in practice with our algorithm. However, the probabilities of such runs can be neglected. [4] gives more details on this.
} 
Fanout. Figure 2 shows the relation between $F$ and the number of rounds it takes to broadcast an event to a system composed of $n=125$ processes. The figure shows that increasing the fanout decreases the number of rounds necessary to infect all processes, but conveys also the fact that the gain is not proportional. In fact, with a too high fanout, there will be more redundant messages received by each process, which limits performance. ${ }^{10}$ Furthermore, $F$ is in our case tightly bound, since $F \leq l$ must always be ensured. The goal of this paper however is not to focus on finding the optimal value for $F .{ }^{11}$ Hence in the following simulations and measurements, the default value for the fanout will be fixed to $F=3$.

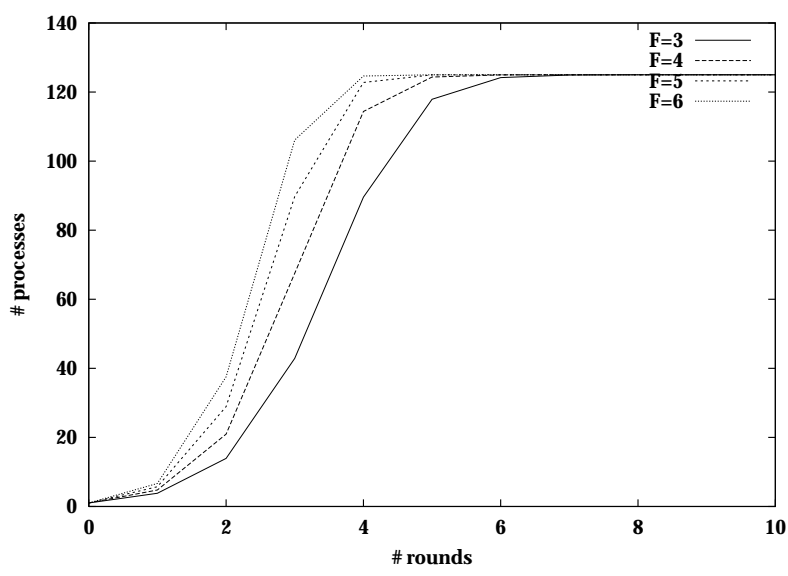

Figure 2. Analysis: expected number of infected processes for a given round with different fanout values

System size $n$. The number of gossip rounds it takes to infect all processes intuitively depends on the number of processes in the system. This is illustrated in Figure 3(a), which depicts the expected number of infected processes for a given round. Figure 3(b) summarizes the expected number of rounds necessary for different system sizes. The figure conveys the fact that the number of rounds increases logarithmically with an increasing system size, as detailed in [3].

View size $l$. According to equation 2, the view size $l$ is not decisive for the time it takes for a notification to reach every member. This leads to the conclusion that, besides the condition $F \leq l$, the amount of knowledge concerning the membership that each process maintains does not have an impact on the protocol performance. The expected number of rounds it takes to infect the entire system depends on $F$, but not on $l$. This consequence derives directly from our assumption that the individual views are uniform. The algorithm shown in Figure 1(b) intuitively supports this hypothesis by two properties, namely (1) each process periodically gossips, and (2) each process adds its own identity to each gossip message. Based on experimental results, we will discuss the validity and impact of this assumption more in detail in Sections 5 and 6.

\footnotetext{
${ }^{10}$ In practice the network would also drop more messages.

${ }^{11}$ This subject is discussed within a different context in [17].
} 


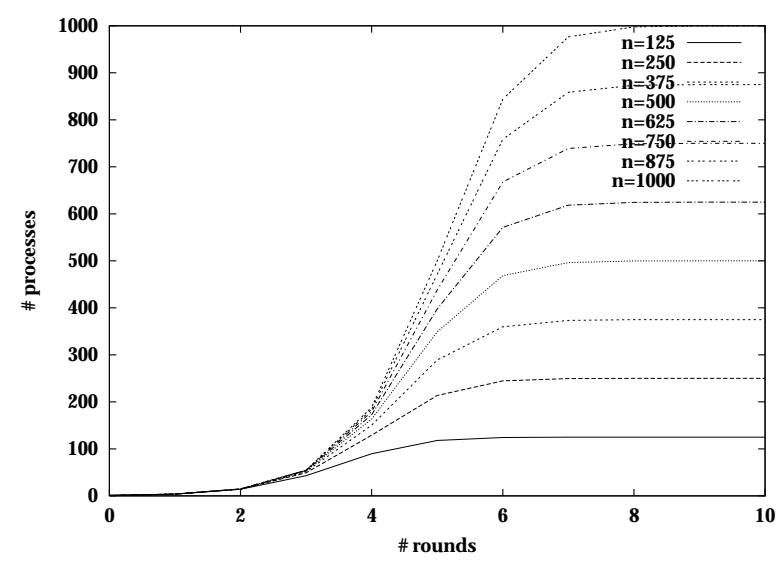

(a) Expected number of infected processes for a given round

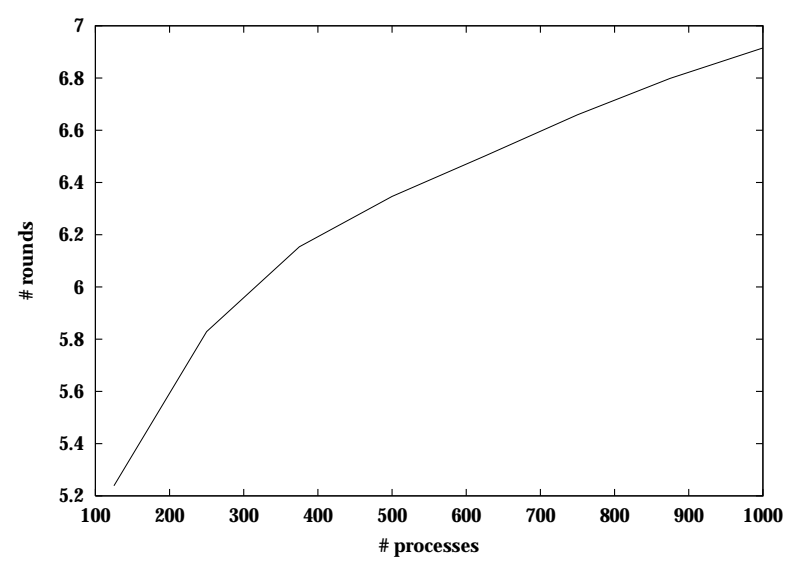

(b) Expected number of rounds necessary to infect $99 \%$ of $\Pi$

Figure 3. Analysis: relation between $l$ and latency

\subsection{Partitioning}

One could derive that $l$ can be chosen arbitrarily small (provided that the requirements with respect to $F$ are met), which is rather dangerous, since with small values for $l$ the probability of system partitioning increases. This occurs whenever there are two or more distinct subsets of processes in the system, in each of which no process knows about any process outside its partition.

Probability of partitioning. The creation of a multiple partition can be seen as a recursive partitioning. In other terms, by expressing an upper bound on the probability of creation of a partition of size $i(i \geq l+1)$ inside the system, we include also the creation of more than two subsets:

$$
\Psi(i, n, l)=\left(\begin{array}{c}
n \\
i
\end{array}\right)\left(\frac{\left(\begin{array}{c}
i-1 \\
l
\end{array}\right)}{\left(\begin{array}{c}
n-1 \\
l
\end{array}\right)}\right)^{i}\left(\frac{\left(\begin{array}{c}
n-i-1 \\
l
\end{array}\right)}{\left(\begin{array}{c}
n-1 \\
l
\end{array}\right)}\right)^{n-i}
$$

It can easily be shown that $\Psi(i, n, l)$ monotonically decreases when increasing $n$ or $l$. Figure 4 depicts this for $n$, by fixing $l$ to 3 . The fact that the membership becomes more stable with an increased $n$ can be intuitively reproduced since, with a large system, membership information becomes more sparsely distributed, and the probability of having concentrated exclusive knowledge becomes vanishingly small.

In time. According to our model, the distribution of membership information in a certain round does not depend on the distribution in the previous round. Thus we can define the probability that there is no partitioning up to a given round $r$ as: 


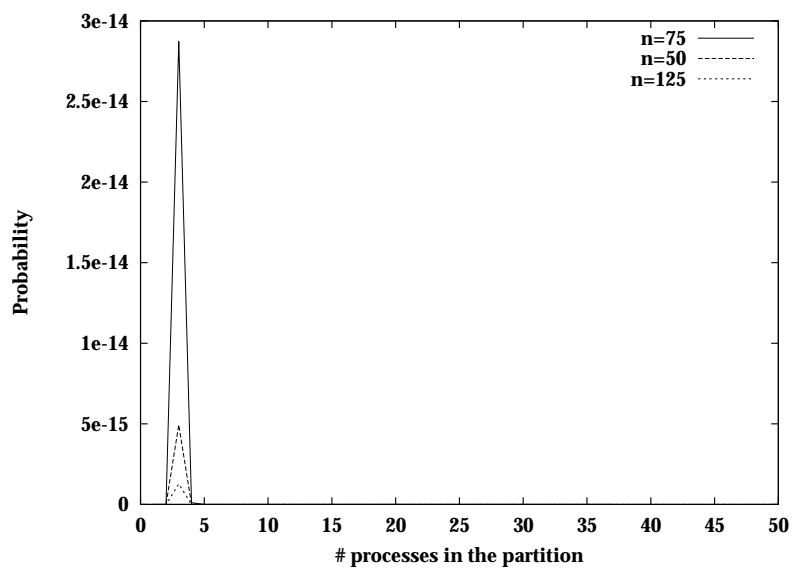

Figure 4. Analysis: probability of partitioning in systems of different sizes

$$
\begin{aligned}
\phi(n, l, r) & =\left(1-\sum_{l+1 \leq i \leq\lfloor n / 2\rfloor} \Psi(i, n, l)\right)^{r} \\
& \approx 1-r \cdot \sum_{l+1 \leq i \leq\lfloor n / 2\rfloor} \Psi(i, n, l)
\end{aligned}
$$

This probability decreases very slowly with $r$. It takes $\approx 10^{12}$ rounds to end up with a partitioned system with a probability of 0.9 with $n=50$ and $l=3$.

A priori, it is not possible to recover from such a partition. To avoid this situation in practice, we elect a very limited set of prioritary processes, which are constantly known by each process. They are periodically used to "normalize" the views (and also for bootstrapping). Alternatively, we could use a set of dedicated processes to collaborate in keeping track of the total number of processes.

\section{Practical Results}

In this section, we compare the analytical results obtained in the previous section with (1) simulation results and (2) results collected from measurements obtained with our actual implementations. In short, the results show a very weak dependency between $l$ and the degree of reliability achieved by lpbcast, but show also that this dependency can be neglected in a practical context.

\subsection{Simulation}

In a first attempt we have simulated the entire system in a single process. More precisely, we have simulated synchronous gossip rounds in which each process gossips once. The results obtained from these simulations support the validity of our analysis. 
Number of gossip rounds. As highlighted in the previous section, the total number of processes $n$ has an impact on the number of gossip rounds it takes to infect all processes. Figure 5(a) conveys the results obtained from our analysis by comparing them with values obtained from simulation, showing a very good correlation.

Impact of $l$. According to the analysis presented in the previous section, the size $l$ of the individual views on the other hand has no impact on the number of gossip rounds it takes to infect every process in the system. Figure 5(b) reports the simulation results obtained for different values for $l$ in a system of 125 processes. It conveys a certain dependency between $l$ and the number of gossip rounds required for the successful dissemination of an event in $\Pi$, slightly contradicting our analysis. This stems from the fact that we have presupposed uniform views for the analysis, and have considered these as completely independent of any "state" of the system. A more precise analysis would have to take into account the exact composition of the view of each process at each round. This would however lead to a very complex Markov Chain, manifesting an impracticable dimension. Given the very good correlation between simulation and analysis, assuming independent and uniform views seems reasonable.

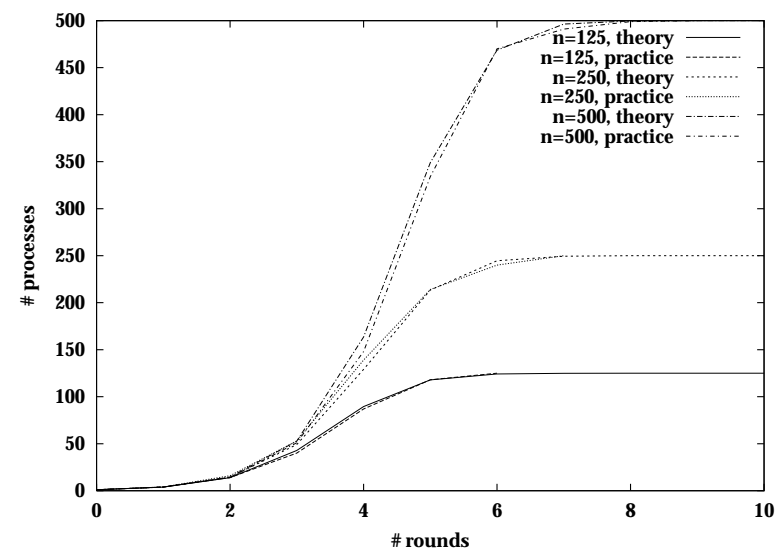

(a) Analysis vs simulation

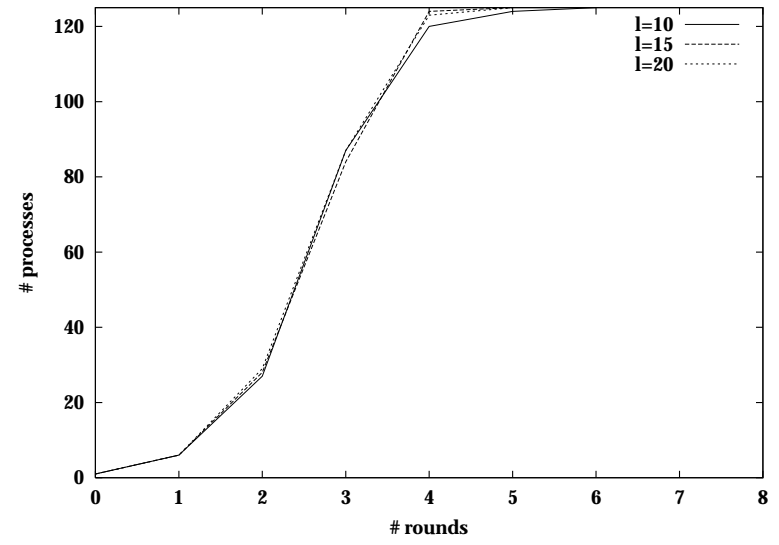

(b) Number of rounds necessary to infect a system with different values for $l$

Figure 5. Simulation results

\subsection{Measurements}

We present here concrete measurements that attempt to capture the degree of reliability achieved with our algorithm, and confirm the results obtained from simulation.

Test environment. Our measurements involved two LANs with 60, respectively 65 SUN Ultra 10 (Solaris 2.6, 256 Mb RAM, 9 Gb harddisk) workstations. The individual stations and the different networks where communicating via Fast Ethernet (100Mbit/s). The measurements we present here were obtained with all 125 processes; each publishing 40 events per gossip round. To conform to our simulations, $F$ was fixed to 3. 
In our test runs, we did not consider retransmissions, that is, once a gossip receiver has received the identifier of a notification, the notification itself is assumed to have been received. This has been done to comply with related work (in some cases it is sufficient for the application to know that it has missed some message(s), and in other cases, subsequent messages can replace the missed messages [23]).

Impact of maximum sizes. Figure 6(a) shows the impact of $l$ on the degree of reliability achieved by our algorithm. The measure of reliability is expressed here by the probability for any given process to deliver any given notification $(1-\beta$, cf. Section 2$)$. The reliability of the system seems to deteriorate slightly with a decreasing value for $l$. Intuitively this seems understandable, since our simulation results have already shown that latency does increase slightly by decreasing $l$. And with an increased latency, the probability that a given message is purged from all buffers before all processes have been infected becomes higher.

With buffers for notifications of infinite length, as we have supposed in the analysis, reliability would remain constant as $l$ becomes smaller. In fact, every notification would be received by every process eventually. In Figure 6(b) we show the strong impact of $\mid$ eventIds $\left.\right|_{m}$ on the reliability of the system $(l=15)$. A more precise expression of the delivery reliability would thus furthermore depend on $l, n$, and $\mid$ events $\left.\right|_{m}$, but also on $|s u b s|_{m}$ and $|u n S u b s|_{m}{ }^{12}$

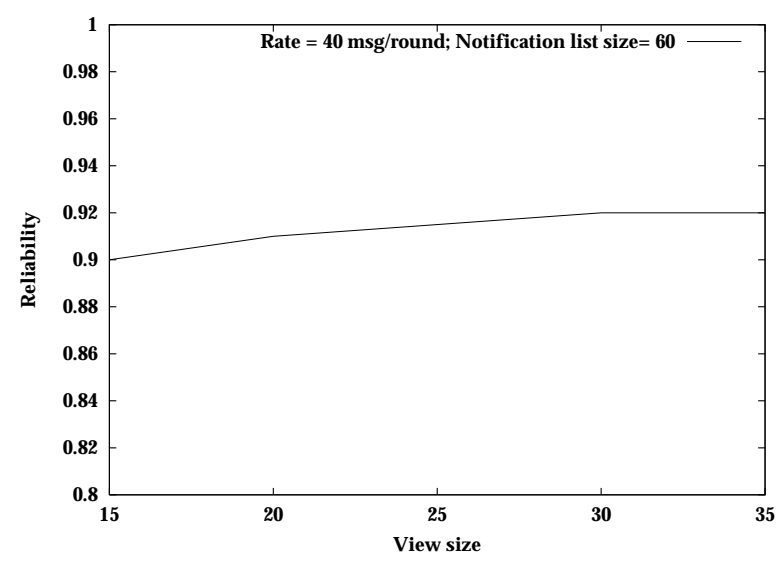

(a) Impact of $l$ on delivery reliability

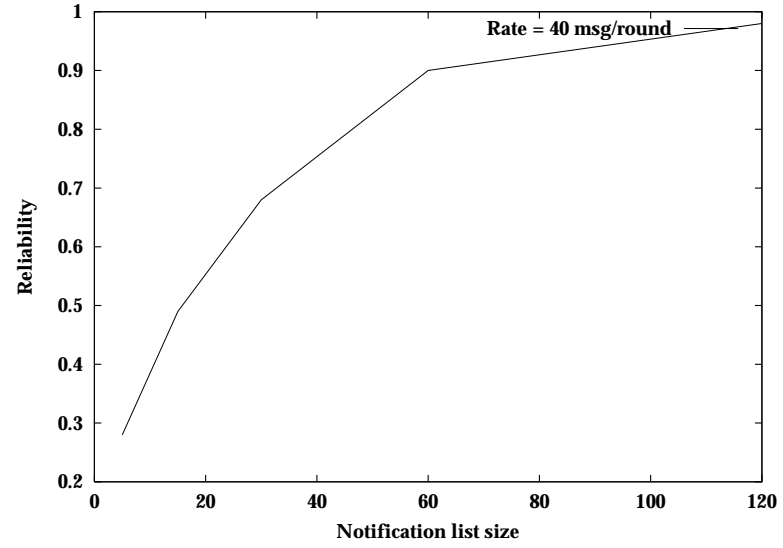

(b) Impact of $\mid$ eventIds $\left.\right|_{m}$ on delivery reliability

Figure 6. Measurements: degree of reliability

As conveyed by our results, the variation in terms of reliability is only very weak, proving the practicability of our algorithm and confirming the validity of our analysis to a large extent. As illustrated here, establishing highly accurate analytical predictions of performance remains a difficult task, given the multitude of involved parameters.

\footnotetext{
${ }^{12}$ Such parameters are hardly ever taken into consideration during the analysis of broadcast algorithms.
} 


\section{Discussion}

This section discusses our lpbcast algorithm with respect to "perfectly" uniform views and compares it closer with the well-known pbcast algorithm [4], in particular by combining pbcast with our membership approach.

\subsection{Towards "Perfect" Views}

Simulations performed with artificially generated independent uniform views have shown that there is virtually no dependency between latency of delivery (and thus the degree of reliability) and the size of the individual views. The views obtained in practice with lpbcast thus are not completely uniform and independant.

Dependency. One interpretation of the slight dependency between latency and $l$ is that, despite the random truncating of views, there remains a correlation between individual views both in time (view ${ }_{i}$ of process $p_{i}$ at round $r$ depends on $v_{i e w_{i}}$ at round $\left.r-1\right)$ and in space $\left(v i e w_{i}\right.$ of process $p_{i}$ depends on view $w_{j}$ of process $\left.p_{j}\right)$. Intuitively, such dependencies negatively affect the latency of delivery of an event: since every process appends part of its view to each outgoing gossip message, a process $p_{i}$, which receives a gossip message from $p_{j}$, has a certain probability of gossiping to processes that have received the same gossip message from $p_{j}$ ( $p_{i}$ updates its view according to the subs it received from $p_{j}$, possibly including processes which have been gossiped to in the same round by $p_{j}$ ).

To avoid this effect, we have tried in a first attempt to reduce the frequency for the gossiping of membership information (every $k$-th round only, $k>1$ ). It has however turned out that this sanction leads to the opposite effect, i.e., latency increases (and thus reliability decreases) further. In contrast, when the frequency for membership gossiping is increased (gossiping membership information more often that events), the views appear to come closer to ideal views, and the performance of our algorithm improves. This is however difficult to apply as an optimization, since $T$ is usually chosen already very small to ensure a high throughput.

Weighted views. As already mentioned, every process should ideally be known by exactly $l$ other processes. This is however difficult to ensure without relying on any form of agreement or counting. We propose an optimization to get the distribution of views closer to this ideal case. It consists in adding a weight to every entry in the view, which gives a measure about how well a given process is known. Unlike the weights used in Directional Gossip [18] however, which represent the connectivity of processes, weights in our case represent the level of awareness for a given process.

When a process $p_{i}$ learns about another process $p_{j}$ which is in $p_{i}$ 's view through the $s u b s$ attached to an incoming gossip message, the weight of $p_{j}$ is increased. When truncating the view, a simple heuristic is applied, consisting in removing entries with a high weight, since these are more probable of being known by many other processes. Furthermore, when constructing subs, a process preferably adds entries from its view with a small weight. A similar scheme could also be applied to events and eventIds.

\subsection{Comparison with pbcast}

Aside from the membership scheme, the main differences between our lpbcast algorithm and pbcast is (1) that the latter algorithm limits the number of hops as well as (2) repetitions for a given message, and (3) that our approach 
melts the two phases of pbcast (dissemination of events, resp. exchange of digests) into a single phase. We comment here on the integration of pbcast with our membership approach, and compare it with our lpcast algorithm.

Membership layer. We have presented our membership approach as integral part of our lpbcast algorithm to ease presentation. As we have already mentioned earlier, our membership approach is nevertheless not inherently coupled with our lpbcast algorithm, but can be separated from the event dissemination process. It could thus be encapsulated as a membership layer, on top of which many gossip-based algorithms, like pbcast, could be deployed. It would act by adding membership information to gossip messages, and would provide quasi-independent uniformly distributed views. Since gossip-based protocols require a random subset of the system, theoretically the size of the view does not impact the probability of infection and hence throughput and delivery latency of the broadcast algorithm would remain virtually unaffected.

Evalation. We simulated the behaviour of a pbcast version instrumented with our membership approach. Figure 7(a) illustrates the process of an event propagation in such a partial view membership for pbcast and lpbcast, comparing with the original pbcast based on a complete view. The advantage of our lpbcast over pbcast can be explained by the fact that hops and repetitions are not limited with the former algorithm.

Figure 7(b) presents the reliability degree measured with different values for $l$ (in every round, each of $n=125$ processes published 40 events). The results are similar to the ones obtained with lpbcast (Figure 6(a)). A direct comparison of the two algorithms is however not a useful measure, since there are different parameters involved. In fact, because repetitions and hops are limited in the case of pbcast, a higher fanout is required to obtain similar results than with lpbcast ( $F=5$ here vs $F=3$ in Figure 6(a)). In fact, lpbcast reaches a higher reliability degree when simulated in the same setting, since its latency is smaller.

In practice and at a high load of the system however, performance can be expected to drop faster with lpbcast, since the first phase of pbcast ensures a high throughput, while gossip messages in lpbcast will transport large numbers of notifications, which might become a bottleneck.

\section{Concluding Remarks}

Gossip protocols have become very attractive for large scale information dissemination because of their nice combination of scalability and reliability. They seem to constitute ideal candidates to support emerging peer-to-peer applications. Though the reliability guarantees they offer are weaker than traditional ones ([14]), the degree of reliability is quantifiable and still very satisfactory in a practical context. In return, gossip protocols excel in terms of scalability. As stated in [19], gossip protocols are scalable because each process sends only a fixed number of messages, and they achieve fault-tolerance because a process receives copies of a message from several processes.

This paper argues for a pragmatic approach where the membership is handled similarly: its scalability is enforced because a process only knows a fixed number of processes, and fault-tolerance can be preserved if each process is known by several processes. This idea is intuitively supported by the fact that gossip messages are only sent to a fixed number of processes. 


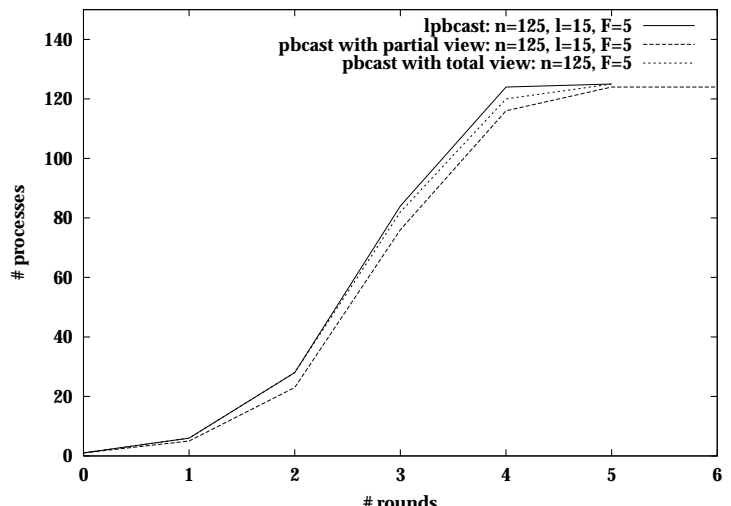

(a) Comparison: number of infected processes in a given round

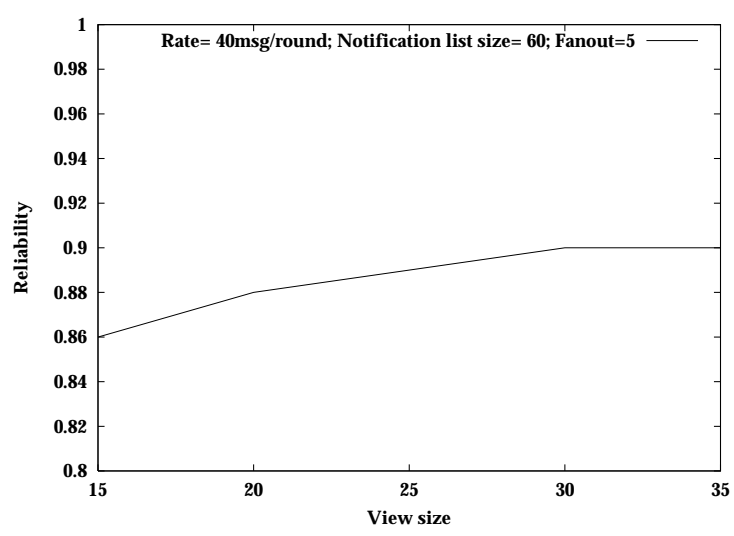

(b) Delivery reliability of pbcast with a random partial view

Figure 7. Simulations and measurements with pbcast

Besides the excellent scalability properties of our Lightweight Probabilistic Broadcast algorithm, we have shown that, in practice, there is very little dependency between its reliability and the size of the views, and this view size can be very small compared to the total size of the system. Giving a precise analytical expression to determine the ideal view size $l$ for a given number of processes and a desired degree of reliability is a hard issue which we are still pursuing. For the time being, the analytical approach we have given here can be used as a tool to tune the algorithm for a given expected maximum system size.

As we have illustrated in this paper through the example of pbcast, our membership approach is not limited to the use with our lpbcast algorithm, but can be put to work easily with other algorithms. We are indeed currently investigating how to combine our membership approach with other gossip-based event dissemination algorithms, e.g., using loggers to ensure strong reliability guarantees whenever this is required (cf. rpbcast).

\section{Acknowledgements}

We are very grateful to Ken Birman and Robert van Renesse for affording us an insight into the subtle approach of probabilistic reliable broadcast.

\section{References}

[1] M. Aguilera, R. Strom, D. Sturman, M. Astley, and T. Chandra. Matching events in a content-based subscription system. In Proceedings of the 18th ACM Symposium on Principles of Distributed Computing (PODC '99), Nov. 1998.

[2] Y. Amir, D. Dolev, S. Kramer, and D. Mahlki. Membership algoritms for multicast communication groups. In 6th Intl. Workshop on Distributed Algorithms proceedings (WDAG), pages 292-312, Nov. 1992.

[3] N. Bailey. The Mathematical Theory of Infectious Diseases and its Applications (second edition). Hafner Press, 1975. 
[4] K. Birman, M. Hayden, O.Ozkasap, Z. Xiao, M. Budiu, and Y. Minsky. Bimodal multicast. ACM Transactions on Computer Systems, 17(2):41-88, May 1999.

[5] A. Carzaniga. Architectures for an Event Notification Service Scalable to Wide-area Networks. PhD thesis, Politecnico di Milano, Dec. 1998.

[6] S. Deering. Internet multicasting. In ARPA HPCC 94 Symposium. Advanced Research Projects Agency Computing Systems Technology Office, Mar. 1994.

[7] A. Demers, D. Greene, C. Hauser, W. Irish, J. Larson, S. Shenker, H. Sturgis, D. Swinehart, and D. Terry. Epidemic algorithms for replicated database maintenance. In Proceedings of the 6th Annual ACM Symposium on Principles of Distributed Computing (PODC'87), pages 1-12, Aug. 1987.

[8] P. Eugster, R. Guerraoui, and J. Sventek. Distributed Asynchronous Collections: Abstractions for publish/subscribe interaction. In Proceedings of the 14th European Conference on Object-Oriented Programming (ECOOP '2000), pages 252-276, June 2000.

[9] C. Fetzer. Fail-aware publish/subscribe in erlang. In Proceedings of the Fourth International Erlang User Conference, Sept. 1998.

[10] S. Floyd, V. Jacobson, S. McCanne, C. G. Liu, and L. Zhang. A reliable multicast framework for light-weight sessions and application level framing. IEEE/ACM Transactions on Networking, Nov. 1996.

[11] R. Golding. Weak-consistency group communication and membership. PhD thesis, University of California at Santa Cruz, Dec. 1992.

[12] Groove Networks, Introducing Groove. http://www.groovenetworks.com/, 2000.

[13] K. Guo, M. Hayden, R. van Renesse, W. Vogels, and K. Birman. GSGC: An efficient gossip-style garbage collection scheme for scalable reliable multicast. Technical Report TR97-1656, Cornell University, Computer Science, Dec. 1997.

[14] V. Hadzilacos and S. Toueg. Distributed Systems, chapter 5: Fault-Tolerant Broadcasts and Related Problems, pages 97-145. Addison-Wesley, 2nd edition, 1993.

[15] M. Hiltunen and R. Schlichting. Properties of membership. In Proceedings of the 2nd IEEE Symposium on Autonomous Decentralized Systems, pages 200-207, Apr. 1995.

[16] H. Holbrook, S. Singhal, and D. Cheriton. Log-based receiver-reliable multicast for distributed interactive simulation. In Proceedings of the 1995 ACM Conference on Applications, Technologies, Architectures, and Protocols for Computer Communication (SIGCOMM '95), pages 328-341, Aug. 1995.

[17] A.-M. Kermarrec, L. Massoulie, and A. Ganesh. Reliable probabilistic communication in large-scale information dissemination systems. Technical Report MSR-TR-2000-105, Microsoft Research Cambridge, Oct. 2000.

[18] M.-J. Lin and K. Marzullo. Directional gossip: Gossip in a wide area network. Technical Report CS1999-0622, University of California, San Diego, Computer Science and Engineering, June 1999.

[19] M.-J. Lin, K. Marzullo, and S. Masini. Gossip versus deterministic flooding: Low message overhead and high reliability for broadcasting on small networks. Technical Report CS1999-0637, University of California, San Diego, Computer Science and Engineering, 1999.

[20] A. Mathur, R. Hall, F. Jahanian, A. Prakash, and C. Rasmussen. The publish/subscribe paradigm for scalable group collaboration systems. Technical Report CSE-TR-270-95, University of Michigan, EECS Department, 1995.

[21] L. Moser, P. Melliar-Smith, and V. Agrawala. Membership algorithms for asynchronous distributed systems. In Proceedings of the 11th International Conference on Distributed Computing Systems, pages 480-489, May 1991. 
[22] L. Opyrchal, M. Astley, J. Auerbach, G. Banavar, R. Strom, and D. Sturman. Exploiting IP Multicast in content-based publish-subscribe systems. In Proceedings of the IFIP/ACM International Conference on Distributed Systems Platforms (Middleware 2000), pages 185-207, Apr. 2000.

[23] J. Orlando, L. Rodrigues, and R. Oliveira. Semantically reliable multicast protocols. In Proceedings of the 19th IEEE Symposium on Reliable Distributed Systems (SRDS 2000), Oct. 2000.

[24] S. Paul, K. Sabnani, J. Lin, and S. Bhattacharyya. Reliable multicast transport protocol (RMTP). IEEE Journal on Selected Areas in Communications, 15(3):407-421, Apr. 1997.

[25] R. Piantoni and C. Stancescu. Implementing the swiss exchange trading system. In Proceedings of The Twenty-Seventh Annual International Symposium on Fault-Tolerant Computing (FTCS '97), pages 309-313, June 1997.

[26] Q. Sun and D. Sturman. A gossip-based reliable multicast for large-scale high-throughput applications. In Proceedings of the IEEE International Conference on Dependable Systems and Networks (DSN2000), New York, USA, July 2000.

[27] TIBCO. TIB/Rendezvous White Paper. http://www.rv.tibco.com/, 1999.

[28] R. van Renesse. Scalable and secure resource location. In Proceedings of the IEEE Hawaii International Conference on System Sciences, 2000.

[29] R. van Renesse, Y. Minsky, and M. Hayden. A gossip-style failure detection service. In Proceedings of the IFIP International Conference on Distributed Systems Platforms and Open Distributed Processing (Middleware '98), Sept. 1998.

[30] Wego.com Inc., What Is Gnutella? http://gnutella.wego.com/, 2000.

\section{Appendix A: Simplification}

Suppose the random variable $\Delta(i)$, which represents the number of processes infected after the current gossip round if $i$ processes are already infected at the beginning of that round. We are interested in predicting the expected value for $\Delta(i)$, which is $E(\Delta(i))$ :

$$
\begin{aligned}
E(\Delta(i))= & \sum_{i \leq j \leq n}(j-i)\left(\begin{array}{c}
n-i \\
j-i
\end{array}\right)\left(1-q^{i}\right)^{j-i}\left(q^{i}\right)^{n-j} \\
= & \sum_{0 \leq j \leq n-i} j\left(\begin{array}{c}
n-i \\
j
\end{array}\right)\left(1-q^{i}\right)^{j}\left(q^{i}\right)^{n-i-j} \\
& \left(\text { expected value of a binomial variable of parameters } n-i \text { and } 1-q^{i}\right) \\
= & (n-i)\left(1-q^{i}\right)
\end{aligned}
$$

And thus the expected value for the number of infected processes $j(i)$ after the next gossip round can be expressed in the following way:

$$
\begin{aligned}
E(j(i)) & =i+E(\Delta(i)) \\
& =i+(n-i)\left(1-q^{i}\right) \\
& =n-(n-i) q^{i}
\end{aligned}
$$

By doing this recursively $t$ times, one can compute the expected number of infected processes after $t$ rounds. Note that at each step, the obtained value might be non-integer, and thus must be rounded off. 